\section{Internetforschung und Techniksoziologie - neue Verknüpfungsnotwendigkeiten durch forcierte Digitalisierung?}

\author{
Bericht von der Jahrestagung der \\ Gesellschaft für Wissenschafts- und \\ Technikforschung (GWTF)
}

Berlin, 20.-21. November 2015

\section{von Stefan Böschen, Institut für Technikfol- genabschätzung und Systemanalyse (ITAS), Karlsruhe}

Am 20. und 21. November 2015 fand die Tagung „Das Internet als Instrument, Infrastruktur oder Institution? Oder: Was die Technikforschung und die Internetforschung voneinander lernen können" in Berlin statt. Die Verknüpfung dieser beiden Forschungsstränge bedarf eigentlich keiner besonderen Begründung, wenn man sich Aktualität und Relevanz von Themen wie „Big Data“, „Open Source“ oder „Industrie 4.0" vor Augen führt. In all diesen erscheint der Bezug zur digitalen Welt des Internet von zentraler Bedeutung - und zugleich erscheinen sie wie Siegel der Gegenwart. Vor diesem Hintergrund stellt sich die Frage der Verknüpfung von Technik- und Internetforschung mit neuer Dringlichkeit.

Einleitend verdeutlichte Christian Stegbauer (Frankfurt a. M.), wie das Internet zunächst als Hoffnungsträger einer egalitären Utopie rangierte, sich aber in der Zwischenzeit verschiedene Phänomene der Ungleichheitsproduktion abzeichneten. So neige die Internetindustrie zur Monopolbildung, was an den Giganten Google oder Apple aufgezeigt wurde: Der Bruttogewinn von Apple entsprach im Jahr 2014 mit 72 Mrd. Euro etwa der Marktkapitalisierung von Siemens, einem der größten DAX-Unternehmen. Das Internet schaffe als Verknüpfungstechnologie ganz unterschiedliche Einbindungsformen und -strukturen, was die Forschung vor neue Herausforderungen stelle. Speziell für die Analyse nichtreaktiver Daten im Internet, also die Datenspuren die jeder Internetnutzer so oder so hinterlässt, bedürfe es einer gesonderten sozialwissenschaftlich aufgeklärten Methodenentwicklung. Manfred Mai (Duisburg) lenkte mit seinen Überlegungen zu „Medium oder großtechnisches System - das Internet zwischen zwei Paradigmen“ den Blick auf den Umstand, dass das Internet ein besonderes großtechnisches System (GTS) sei. Im Gegensatz zu herkömmlichen Formen, die durch Gebietsmonopolisten (wie RWE im Fall des Energiesystems), eine starke Rolle des Staates und einen regionalen Bezug geprägt seien, sei das Internet gekennzeichnet durch Unternehmensgiganten und eine generell globale, tendenziell wenig vom Staat regulierte Entwicklungsdynamik. Zugleich verfüge es über Qualitäten eines Massenmediums mit einer Vielfalt von Netzwerkoptionen, kreativen Aneignungsformen und verschiedenen soziokulturellen Szenen. $\mathrm{Zu}$ sammen genommen präsentiere sich das Internet als ein GTS mit einer besonders ausgeprägten Flexibilität. Als Thesen stellte Manfred Mai in den Raum: Internet und andere GTS verschmelzen gegenwärtig zu einem Super-GTS (man denke an Industrie 4.0). Durch diese Verschmelzung würden herkömmliche GTS zwar flexibler aber auch verletzlicher. Schließlich sei es offen, wer wen dominieren werde: die Internetökonomie die Industrieökonomie oder umgekehrt.

\section{Open Source}

Im ersten Themenblock „Open Source“ argumentierte Jan-Felix Schrape (Stuttgart) mit seinen Überlegungen „Open Source Projekte zwischen Passion und Kalkül“" gegen eine zu rasche Fokussierung auf die Selbstbeschreibung der InternetCommunities. Dort gehe man von einer radikal dezentralisierten ,peer-production“ aus, die unter dem Schlagwort Open Source selbst zu einem Label geworden sei. Jedoch konnte Schrape überzeugend darlegen, dass es ganz unterschiedliche Typen der Einbindung von Peers in Software-Projekte geben kann. In diesem Sinne unterschied er anhand der beiden Dimensionen Unternehmenseinfluss (hoch/niedrig) und Koordination (hierarchisch/heterarchisch) vier Varianten und unterlegte diese mit empirischen Beispielen. Die beiden weiteren Vorträge dieser Session von David Schünemann (Oldenburg) ,,Social Coding ${ }^{6}$ - Zur Verschränkung von Institutionen und Technik in FOSS-Gemeinschaften" sowie Daniel Guagnin (Berlin) ,Zwischen Selbstbestimmung und Meri- 
tokratie. Freie-Software Gemeinschaften als soziotechnische epistemische Regime" untersuchten die Besonderheiten von Gemeinschaften der Software-Produktion hinsichtlich des Merkmals Identität und stellten die Frage nach der spezifischen Teilung und Produktion von Wissen sowie den dabei konstitutiven Regeln nach, sodass diese als Wissensregime beschrieben werden könnten.

\section{Algorithmen}

Den zweiten Themenblock zu „Algorithmen“, eröffneten Katharina Kinder-Kurlanda und JanHinrik Schmidt (Köln/Hamburg) mit ihrem Beitrag zu „Rekursivität und Algorithmen: Big Data's Moving Targets". Die Spannung in diesem Thema ergibt sich durch die Besonderheit algorithmischer Öffentlichkeiten, die aufgrund der technisierten Vermittlung eigenen Logiken folgen. Die Verdatung und Algorithmisierung transformieren soziale Phänomene, um sie zugleich auch Praktiken des Vermessens zugänglich zu machen. Deshalb stellen sich Fragen von Macht und Kontrolle. Zugleich kann sich aber der Nutzer reflexiv dazu verhalten. Zwei Modi wurden dabei benannt: Erstens die rekursive Verstärkung, bei der es zu einer Konzentration von Aufmerksamkeit kommt, die auch noch technisch durch Plattformen oder ,filter bubbles" (Eli Pariser) verstärkt werden kann. Bei diesen werden Teilöffentlichkeiten durch personalisierte Merkmale der Internetnutzung gebildet, d. h. Menschen werden von der Vielfalt von Standpunkten und Inhalten gezielt isoliert und die Konfrontation mit dissonanten Inhalten verringert. Zweitens die strategische Koordination, welche von den Vortragenden als ,gaming the algorithm“ charakterisiert wurde. Das heißt, dass spezifische Merkmale von Plattformen, wie das „Like“, strategisch dazu genutzt werden können, Informationen gegenüber anderen stärker hervortreten zu lassen. Ihre Schlussfolgerung: Zwar würden Designentscheidungen die Rahmenbedingungen für das dynamische Wechselspiel zwischen Algorithmus und Daten schaffen, zugleich könnten Plattformbetreiber die Dynamik nur begrenzt kontrollieren. Sicherlich würden Algorithmen unter Regulierungsdruck geraten, aber zugespitzt stellt sich die komplementäre Frage, ob Rekursivität reguliert werden kann. In der Diskussion wurde herausge- stellt, dass der Zusammenhang von Algorithmus und Relevanzzuschreibung in sich vielschichtiger sei, da er als wirksame Trias von Algorithmen, Designern und Nutzern konzeptualisiert werden müsste. Dieser Punkt wurde von Gernot Grabher und Jonas König (Hamburg) mit ihrem Beitrag zu „Performing Network Theory? Social Networking Sites und die Praktiken professioneller NetzwerkBildung" noch unterstrichen. Unter Bezug auf die Vorstellung einer "socio-cognitive prosthesis“ von Technologien und Algorithmen fokussierten sie auf Phänomene der Performativität. Gerade weil die Interaktion zwischen Algorithmen und Akteuren nicht linear, sondern eher rekursiv sei, sollte man das Internet performativ betrachten. Generische Performativität (i. S. v. Callon) meint, dass das Internet nicht einfach gegeben sei, sondern sich in der Durchführung von Internetpraktiken realisiere. Neben dieser prinzipiellen Form von Performativität weitere zu untersuchen, das sei für die Forschungsagenda relevant.

\section{Internetforschung und Techniksoziologie}

In dem Perspektiven generierenden Abendvortrag entwickelte Ulrich Dolata (Stuttgart) unter dem Titel „Kollektive Formationen im Internet. Akteurtheoretische und techniksoziologische Zugänge" einen Rahmen für die weitere Theoretisierung des Zusammenhangs von Internetforschung und Techniksoziologie. Seine Argumentation entwickelte eine doppelte Differenzierung, der von kollektiven Formierungen und der von Infrastrukturen, um dann deren Relation zu untersuchen. Kollektive Formierungen differenzierte Dolata in vier Typen: individuelle Akteure, nicht-organisierte Kollektive, kollektive Akteure und korporative Akteure, die er detailliert aus einer handlungstheoretischen Perspektive heraus entwickelte. Der entscheidende Switch geschieht dadurch, dass man die soziotechnische Institutionalisierung solcher kollektiven Formierungen genau mit in den Blick nimmt. Diese lassen sich letztlich als zwei Kontexte erfassen, erstens die Bedeutung technischer Infrastrukturen (mit ihren Binnenstrukturen von Beteiligung, Beobachtung und Transparenz sowie der Außenwirkung von Skandalisierung, Sichtbarkeit und Mobilisierung) sowie zweitens die Ausprägung technischer Infrastrukturen (die 
entweder als privatwirtschaftliche, wie Facebook oder Twitter, oder formationsspezifische Plattformen, wie die Wikipedia, in Erscheinung treten). Diese Plattformen vollbringen spezifische Strukturierungsleistungen und werden durch unterschiedliche institutionelle Elemente konfiguriert. Von daher treffe das Diktum von Popitz „Vom Hersteller in das Ding eingebaute Macht" hier sehr gut. Zusammen genommen präsentierte Dolata einen stimmigen Rahmen von Analysewerkzeugen, der für die künftige techniksoziologische Internetforschung zentral sein wird.

\section{Wissenschaft}

Der dritte Themenblock wandte sich dem Feld Wissenschaft zu. Martina Franzen (Berlin) lotete dabei die „Folgen der digitalen Revolution“ in diesen Bereich aus. Rekurrierend auf eine gesellschaftstheoretische, genauer: kommunikationstheoretische Perspektive, analysierte sie das Internet als Verbreitungsmedium. Zwar können das Web 2.0 als Enabler von Open Science angesehen werden, da neue Optionen der Interaktion zwischen Wissenschaft und etwa der Öffentlichkeit geschaffen würden, zugleich wandelten sich aber auch die Binnenverhältnisse innerhalb der Wissenschaft. Dies lässt sich sehr gut an den Formen wissenschaftlicher Leistungsmessung und ihres Wandels beobachten. Dabei ging Franzen auf die Altmetrics-Bewegung und neue Formen der Impact-Messung ein, die sich am Prinzip „Follow the user" orientierten und in der Public Library of Science (PLOS) einen Vorreiter in der Multiplizierung von Bewertungsdimensionen habe. Im Gegensatz dazu nahmen Monika Taddicken, Nina Wicke, Laura Wolff, Anne Reif und Irene Neverla (Braunschweig/Hamburg) mit ihrem Beitrag ,\#Klimawandel - Neue kommunikative Praktiken auf Twitter in der Wissenschaftskommunikation?" das Verhältnis von Wissenschaft und öffentlicher Kommunikation in den Blick.

\section{Kommunikation und Beteiligung}

Der vierte und letzte Block „Kommunikation und Beteiligung“" wurde von dem stärker kon- zeptionell angelegten Vortrag „Die Grenzen der Internetkommunikation. Zur Verknüpfung technik- und internetsoziologischer Forschung im Rahmen einer userzentrierten Perspektive“ von Christian Papsdorf (Chemnitz) eröffnet. Dabei stellte er die Frage, wie und wo sich Grenzen der Internetkommunikation konstituieren. Die eine Grenze vollziehe sich an der Trennung zwischen online und offline. Zugleich gäbe es aber eine Reihe von sich praktisch vollziehenden Grenzen. Dabei kritisierte Papsdorf die bisherige Debatte zum Digital Divide, in der es immer wieder zu Verkürzungen kommt, wie etwa der NichtBerücksichtigung von freiwilligem Verzicht als Option oder die akteursabhängigen Nutzungserwartungen, welche verdeutlichten, dass die Nutzer als Kategorie eigentümlich marginalisiert seien. Deshalb warb der Referent für das Mediatisierungskonzept, das gegen die Sachvergessenheit der Soziologie stehe. So vertiefte er seine Überlegungen an Problemstellungen der Privacy (Grenze privat/öffentlich) und der konkreten Internetnutzung (körperliche Grenzen beim Nutzen), um solche in der Praxis der Internetnutzung auftauchenden und sich verfestigenden Grenzen zu thematisieren. Es ist also gleichsam das ,doing boundaries" in der jeweiligen Internetpraxis zu analysieren, z. B. die Ermöglichung wie Begrenzung von Partizipation durch die jeweilige Plattformarchitektur. Abschließend führten $\mathrm{Sa}$ scha Dickel, Andrea Geipel, Jan-Hendrik Passoth, Silvan Pollozek, Carolin Thiem und Andreas Wenninger (München) mit ihrem Vortrag „Beteiligungsmaschinerien: Versachlichung als Inklusionschance" ganz verschiedene Settings der Beteiligung vor Augen. Dabei gingen sie von der Beobachtung einer Art „All-Partizipation“ durch das Web 2.0 aus. Dies ginge auf der einen Seite mit der Utopie der Beteiligung, auf der anderen Seite mit der Dystopie einer weitreichenden Versachlichung einher. Anhand unterschiedlicher Settings, die von der Standardisierung in der Medizin bis zu Crowd Science reichten, führten die Vortragenden unterschiedliche Beteiligungsmaschinerien plastisch vor. Da Versachlichung eine Art Infrastruktur modernisierter Gesellschaften darstelle, zeigten sich die Beteiligungsmaschinerien als eine ,partizipative Selbsttäuschung der Moderne" (Passoth). Nach der eindrücklichen 
Darstellung solcher Beteiligungsmaschinerien wurde in der Diskussion die Frage nach gescheiterten Formen von Beteiligung gestellt und was Kriterien des Scheiterns im Lichte der dargestellten Analytik von Utopie und Dystopie seien.

Die Tagung zeigte die Vielfalt möglicher Verknüpfungen zwischen Internetforschung und Techniksoziologie. Sie machte deutlich, wie zentral die Hinwendung zu Problemstellungen der Digitalisierung für die weitere technik- und wissenschaftssoziologische Forschung sein wird, - und dass hierfür das analytische Rüstzeug erst noch entwickelt werden muss. Anregungen dazu hielten die ReferentInnen in vielfältiger Weise bereit.

Das Programm der Tagung findet man unter http://www.gwtf.de/2015-programm-gwtf.pdf.

\section{$《 》$}

\section{Fachportal openTA}

openTA ist das Fachportal des Netzwerks TA (NTA). Es aggregiert Nachrichten, Termine und Publikationen aus dem NTA, gibt einen Überblick über die Personen und Institutionen, die im Bereich der TA in Deutschland, Österreich und der Schweiz tätig sind, und erlaubt eine personalisierte und dezentrale Nutzung (etwa über Feeds, Widgets).

Die folgenden Dienste stehen gegenwärtig zur Verfügung:

Der openTA-Newsdienst informiert tagesaktuell über Projekte, Publikationen, Veranstaltungen und Kooperationsangebote aus zur Zeit zehn Mitgliedsinstitutionen des NTA sowie sechs weiteren Institutionen des europäischen Netzwerks der parlamentarischen TA-Institutionen (EPTA).

Der openTA-Kalenderdienst bietet einen Überblick über die Veranstaltungen der NTA-Institutionen und über weitere Termine mit TARelevanz. Die Termine des openTA-Kalenders lassen sich in eigene Kalenderanwendungen (Outlook, Thunderbird etc.) übernehmen.

Der openTA-Publikationsdienst aggregiert monatlich die Publikationsnachweise aus sieben Mitgliedsorganisationen des NTA sowie aus weiteren TA-relevanten Quellen. Er enthält gegenwärtig fast 25.000 Publikationsnachweise bis in die 1970er Jahre zurück.

Der openTA-Neuerscheinungsdienst filtert aus den Daten der Deutschen Bibliothek monatlich etwa 30 bis 40 aktuelle, besonders interessante Monographien und kommentiert diese Auswahl in einem Beitrag auf dem openTA-Blog unter dem Titel ,überdenTAellerrand“.

Alle diese Dienste können nach bestimmten Kategorien gefiltert und auch im Volltext durchsucht werden.

Das Fachportal openTA wurde aus dem NTA heraus initiiert, von der DFG gefördert und von einem Konsortium des KIT unter Federführung des ITAS realisiert.

Nutzen Sie die Möglichkeit, Ihre Inhalte auch über openTA zu verbreiten! Bitte setzen Sie sich dazu mit uns in Verbindung.

Kontakt: Ulrich Riehm; info@openTA.net

Internet: https://www.openTA.net 